\title{
Charged magnetoexcitons in two-dimensions: Magnetic translations and families of dark states
}

\author{
A. B. Dzyubenkd* \\ Institut für Theoretische Physik, J.W. Goethe-Universität, 60054 Frankfurt, Germany
}

A. Yu. Sivachenko

The Weizmann Institute of Science, Rehovot 76100, Israel

(October 9, 2018)

\begin{abstract}
We show that optical transitions of charged excitons in semiconductor heterostructures are governed in magnetic fields by a novel exact selection rule, a manifestation of magnetic translations. It is shown that the spin-triplet ground state of the quasi-two-dimensional charged exciton $X^{-}-$ a bound state of two electrons and one hole - is optically inactive in photoluminescence at finite magnetic fields. Internal bound-to-bound $X^{-}$triplet transition has a specific spectral position, below the electron cyclotron resonance, and is strictly prohibited in a translationally-invariant system. These results allow one to discriminate between localized and free charged excitons.
\end{abstract}

In quasi-two-dimensional (quasi-2D) electron-hole $(e-h)$ systems with low density of particles, a variety of hydrogenic few-particle complexes can be formed. Optical spectroscopy in magnetic fields $B$ is one of the basic tools for studying such complexes. Recently, much experimental [1]7 and theoretical 8 12] attention has been devoted to studying negatively $X^{-}(2 e-h)$ and positively $X^{+}(2 h-e)$ charged excitons in magnetic fields $B$. These complexes are often considered to be semiconductor analogs of the hydrogen atomic $H^{-}$and molecular $\mathrm{H}_{2}^{+}$ions, respectively. In $B$, in addition to the spinsinglet, higher-lying spin-triplet bound states of $X^{-}$and $X^{+}$develop [1]:2]. The question as to whether these complexes are mobile and free to move, or are localized by single donor impurities [5, [6]10], disorder due to longrange fluctuating potential of remote donors [6] etc. is a matter of current controversy. To explore these issues, we theoretically address from first principles the following question: Are there fundamental differences in optical transitions between mobile and localized charged $e-h$ systems in magnetic fields?

For a one-component translationally-invariant interacting electron system in $B$, the well-known Kohn theorem 13 states that intraband transitions can occur only at the bare electron cyclotron resonance $(e-\mathrm{CR})$ energy $\hbar \omega_{\mathrm{ce}}=\hbar e B / m_{e} c$. This is a consequence of the center-ofmass $(\mathrm{CM})$ separation from internal degrees of freedom in $B$. For $e^{-h}$ systems such separation is not possible and the $\mathrm{CM}$ and internal motions are coupled in $B$ [14, 15. However, for any system of charged particles in a uniform $B$ an exact symmetry - magnetic translations exists ( [15] and references therein). It has been used to study the motion of atoms and ions in constant magnetic and electric fields 16. This symmetry, however, has not been identified in previous theoretical work on charged excitons in $B$. In this work, we introduce for charged semiconductor $e-h$ complexes in $B$ an exact classification of states, which is based on magnetic translations [15]. In this scheme, in addition to the total orbital an- gular momentum projection $M_{z}$ and spin of electrons $S_{e}$ and holes $S_{h}$, an exact quantum number, the oscillator quantum number $k$, appears. Surprisingly, only very general consideration of radiation processes in $B$ using magnetic translations has been given 15]. To our knowledge, no selection rule associated with $k$ has been established for dipole-allowed magneto-optical transitions. We show here that $k$ is strictly conserved in the intraband and in interband magneto-optical transitions. This leads to striking spectroscopic consequences for charged excitons.

Consider a many-body Hamiltonian of interacting particles of charges $e_{i}$ in a magnetic field $\mathbf{B}=(0,0, B)$

$$
H=\sum_{i} \frac{\hat{\pi}_{i}^{2}}{2 m_{i}}+\frac{1}{2} \sum_{i \neq j} U_{i j}\left(\mathbf{r}_{i}-\mathbf{r}_{j}\right)
$$

here $\hat{\boldsymbol{\pi}}_{i}=-i \hbar \boldsymbol{\nabla}_{i}-\frac{e_{i}}{c} \mathbf{A}\left(\mathbf{r}_{i}\right)$ and potentials of interparticle interactions $U_{i j}$ can be rather arbitrary. In the symmetric gauge $\mathbf{A}=\frac{1}{2} \mathbf{B} \times \mathbf{r}$ the total angular momentum projection $M_{z}$, an eigenvalue of $\hat{L}_{z}=\sum_{i}\left(\mathbf{r}_{i} \times-i \hbar \boldsymbol{\nabla}_{i}\right)_{z}$, is an exact quantum number. In a uniform $\mathbf{B}$ the Hamiltonian (11) is also invariant under a group of magnetic translations whose generators are the components of the operator $\hat{\mathbf{K}}=\sum_{i} \hat{\mathbf{K}}_{i}$, where $\hat{\mathbf{K}}_{i}=\hat{\boldsymbol{\pi}}_{i}-\frac{e_{i}}{c} \mathbf{r}_{i} \times \mathbf{B}$ and $\left[\hat{K}_{i p}, \hat{\pi}_{i q}\right]=0, p, q=x, y$ [14 16]. $\hat{\mathbf{K}}$ is an exact integral of the motion $[H, \hat{\mathbf{K}}]=0$. Its components commute as

$$
\left[\hat{K}_{x}, \hat{K}_{y}\right]=-i \frac{\hbar B}{c} Q \quad, \quad Q \equiv \sum_{i} e_{i} .
$$

For neutral complexes (excitons, biexcitons) $Q=0$ and classification of states in $\mathbf{B}$ is due to the two-component continuous vector - the $2 \mathrm{D}$ magnetic momentum $\mathbf{K}=$ $\left(K_{x}, K_{y}\right)$ [14,15]. For charged systems $Q \neq 0$ and the components of $\hat{\mathbf{K}}$ do not commute. This determines the macroscopic Landau degeneracy of eigenstates of (11). Using a dimensionless operator $\hat{\mathbf{k}}=\sqrt{c / \hbar B|Q|} \hat{\mathbf{K}}$ whose components are canonically conjugate, one obtains raising and lowering Bose ladder operators $\hat{k}_{ \pm}=$ 
$\left(\hat{k}_{x} \pm i \hat{k}_{y}\right) / \sqrt{2}$ such that $\left[\hat{k}_{-}, \hat{k}_{+}\right]=Q /|Q|$. Therefore, $\hat{\mathbf{k}}^{2}=\hat{k}_{+} \hat{k}_{-}+\hat{k}_{-} \hat{k}_{+}$has the oscillator eigenvalues $2 k+1$, $k=0,1, \ldots$ Since $\left[\hat{\mathbf{k}}^{2}, H\right]=0$ and $\left[\hat{\mathbf{k}}^{2}, \hat{L}_{z}\right]=0$, the exact charged eigenstates of (11) can be simultaneously labeled by the discrete quantum numbers $k$ and $M_{z}$ [15]. For charged $e^{-} h$ complexes in $B$ the labelling therefore is $\left|k M_{z} S_{e} S_{h} \nu\right\rangle$, where $\nu$ is the "principal" quantum number, which can be discrete (bound states) or continuous (unbound states forming a continuum); concrete examples are given below. The $k=0$ states are Parent States (PS's) within a degenerate manifold. All other daughter states $k=1,2, \ldots$ in each $\nu$-th family can be generated out of the PS: for, e.g., $Q<0$

$$
\left|k, M_{z}-k, S_{e} S_{h} \nu\right\rangle=\frac{1}{\sqrt{k !}} \hat{k}_{-}^{k}\left|0, M_{z}, S_{e} S_{h} \nu\right\rangle,
$$

where we have used $\left[\hat{L}_{z}, \hat{k}_{ \pm}\right]= \pm \hat{k}_{ \pm}$. The values of $M_{z}$ that the PS's have are determined by particulars of interactions and cannot be established a priori (cf. with $2 \mathrm{D}$ electron systems in strong $B$ [17]).

In the dipole approximation the photon momentum is negligibly small. Therefore, the quantum number $k$ should be conserved in intra- and inter-band magnetooptical transitions. Let us establish this selection rule formally. For internal intraband transitions in the Faraday geometry (light propagating along B) the Hamiltonian of the interaction with the radiation of polarization $\sigma^{ \pm}$is of the form $\hat{V}^{ \pm}=\sum_{i}\left(e_{i} \mathcal{F}_{0} \hat{\pi}_{i \pm} / m_{i} \omega\right) e^{-i \omega t}$, where $\mathcal{F}_{0}$ is the radiation electric field, $\hat{\pi}_{i \pm}=\hat{\pi}_{i x} \pm i \hat{\pi}_{i y}$ (e.g., [13). Conservation of $k$ follows from the commutativity $\left[\hat{V}^{ \pm}, \hat{\mathbf{K}}\right]=0$ [18]. (In fact the perturbation $\hat{V}=F\left(\hat{\boldsymbol{\pi}}_{i}, t\right)$ can be an arbitrary function of kinematic momentum operators $\hat{\boldsymbol{\pi}}_{i}$ and time $t$, corresponding, e.g., to other geometries and polarization.) Other usual selection rules are conservation of spins $S_{e}, S_{h}$ and $\Delta M_{z}= \pm 1$ for the envelope function in the $\sigma^{ \pm}$polarization. This means that the PS's should be connected by the dipole transition, i.e., have proper spins and $M_{z}^{\prime}-M_{z}= \pm 1$. Indeed, for the transition dipole matrix element between the daughter states in the $k^{\prime}$-th and $k$-th generations we have

$$
\begin{aligned}
\mathcal{D}_{\nu^{\prime} \nu} & =\left\langle k^{\prime}, M_{z}^{\prime}-k^{\prime}, S_{e} S_{h} \nu^{\prime}\left|\hat{V}^{ \pm}\right| k, M_{z}-k, S_{e} S_{h} \nu\right\rangle \\
& =\delta_{k^{\prime}, k} \delta_{M_{z}^{\prime}, M_{z} \pm 1}\left\langle 0, M_{z}^{\prime}, S_{e} S_{h} \nu^{\prime}\left|\hat{V}^{ \pm}\right| 0, M_{z}, S_{e} S_{h} \nu\right\rangle .
\end{aligned}
$$

Here we have used (3) and the operator algebra $\left[\hat{V}^{ \pm}, \hat{k}_{-}\right]=\left[\hat{V}^{ \pm}, \hat{k}_{+}\right]=0,\left[\hat{k}_{+}, \hat{k}_{-}\right]=1$. From (位) it is clear that $\mathcal{D}_{\nu^{\prime} \nu}$ is the same in all generations and, thus, characterizes the two families of states. Similar considerations apply to interband transitions with $e^{-} h$ pair creation or annihilation: The interaction with the radiation field is described by the luminescence operator $\hat{\mathcal{L}}_{\mathrm{PL}}=p_{\mathrm{cv}} \int d \mathbf{r} \hat{\Psi}_{e}^{\dagger}(\mathbf{r}) \hat{\Psi}_{h}^{\dagger}(\mathbf{r})+$ H.c., where $p_{\mathrm{cv}}$ is the interband momentum matrix element (e.g., [19]). Here we have the commutativity $\left[\hat{\mathcal{L}}_{\mathrm{PL}}, \hat{\mathbf{K}}\right]=0$, so that $k$ is conserved. Due to the change of the Bloch parts in this case, the usual selection rule $\Delta M_{z}=0$ holds for the envelope functions.

Conservation of $k$ constitutes an exact selection rule for the dipole-allowed magneto-optical transitions. It is applicable to any charged $e-h$ system in $B$. Mobile charged excitons $X^{-}, X^{+}$, charged multiple-excitons $X_{N}^{-}$[9, 12], are particular examples. In some limiting cases $k$ can be directly related to the center of the cyclotron motion of the complex as a whole 15,16]. This gives some physical insight into its conservation. In the derivation above we only used translational invariance in the plane perpendicular to B. Therefore, conservation of $k$ holds in arbitrary magnetic fields and for systems of different dimensionality. Importantly, it is also applicable to semiconductors with complex valence band. Indeed, $k$ is a good quantum number for the Luttinger Hamiltonian, while $M_{z}$ is replaced by the combination $\mathcal{M}_{z}=M_{z}+S_{\mathrm{hz}}+S_{\mathrm{ez}}$ involving the $e$ - and $h$ - spin projections; $\mathcal{M}_{z}$ is conserved in the usual axial approximation (e.g., Ref. [19], p. 48).

To make further discussion more concrete, we consider the strictly-2D $e-h$ system in the limit of high $B$ [9, 10], when $\hbar \omega_{\text {ce }}, \hbar \omega_{\text {ch }} \gg E_{0}=\sqrt{\pi / 2} e^{2} / \epsilon l_{B}$ and mixing between Landau levels (LL's) can be neglected; $l_{B}=$ $(\hbar c / e B)^{1 / 2} . E_{0}$ is the characteristic energy of Coulomb interactions, the only energy scale in the problem. The basis for the $X^{-}$states 20] in the electron and hole LL's $\left(N_{e} N_{h}\right)$ is of the form $\phi_{n_{1} m_{1}}^{(e)}\left(\mathbf{r}_{e}\right) \phi_{n_{2} m_{2}}^{(e)}\left(\mathbf{R}_{e}\right) \phi_{N_{h} m_{h}}^{(h)}\left(\mathbf{r}_{h}\right)$ and includes different three-particle $2 e-h$ states such that the total angular momentum projection $M_{z}=n_{1}+n_{2}-$ $m_{1}-m_{2}-N_{h}+m_{h}$, and LL's $N_{e}=n_{1}+n_{2}, N_{h}$ are fixed 21.22. Here $\phi_{n m}^{(e, h)}$ are the $e$ - and $h$ - single-particle factored wave functions in $B$ (e.g., [15, 16]); $n$ is the LL quantum number and $m$ is the single-particle oscillator quantum number $\left(m_{\mathrm{ze}}=-m_{\mathrm{zh}}=n-m\right)$. We use the electron relative $\mathbf{r}_{e}=\left(\mathbf{r}_{e 1}-\mathbf{r}_{e 2}\right) / \sqrt{2}$ and CM $\mathbf{R}_{e}=\left(\mathbf{r}_{e 1}+\mathbf{r}_{e 2}\right) / \sqrt{2}$ coordinates. The electron relative motion angular momentum $n_{1}-m_{1}$ should be even (odd) in the electron spin-singlet $S_{e}=0$ (triplet $S_{e}=1$ ) state. To make this basis compatible with magnetic translations, i.e., to fix $k$, an additional canonical transformation diagonalizing $\hat{\mathbf{k}}^{2}$ should be performed; details will be given elsewhere. The method of analytical calculation of the Coulomb matrix elements has been described in [21,22].

The calculated three-particle $2 e-h$ eigenspectra with electrons in the triplet $S_{e}=1$ state in two lowest LL's are shown in Fig. 1. The spectral properties of the charged three-body problem in strong $B$ is interesting in itself [15,16]. Generally, the eigenspectra associated with each LL consist of bands of finite widths $\sim E_{0}$. The states within each such band form a continuum corresponding to the extended motion of a neutral magnetoexciton (MX) as a whole with the second electron in a scattering state (on average at infinity from the MX). For example, the continuum in the $\left(N_{e} N_{h}\right)=(10)$ LL consists of the MX band of width $E_{0}$ extending down in energy from 


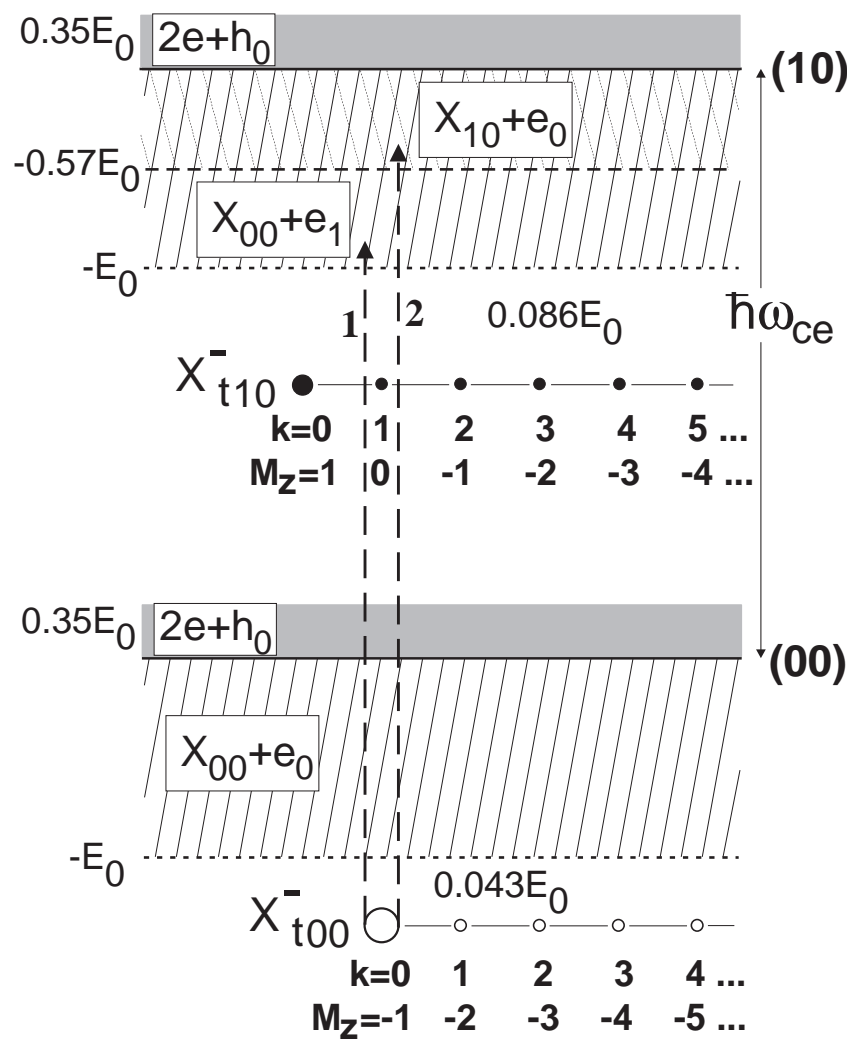

FIG. 1. Schematic drawing of bound and scattering electron triplet $2 e-h$ states in the lowest LL's $\left(N_{e} N_{h}\right)=(00),(10)$. Large (small) dots correspond to the bound parent $k=0$ (daughter $k=1,2, \ldots) X^{-}$states. Allowed internal transitions must satisfy $\Delta M_{z}=1$ and $\Delta k=0$.

the free (10) LL. This corresponds to the $1 s$ MX in zero LL's [23] plus a scattered electron in the first LL, labeled $X_{00}+e_{1}$. (A similar continuum exists in zero LL's.) In addition, there is another MX band of width $0.574 E_{0}$ also extending down in energy from the free (10) LL. This corresponds to the $2 p^{+}$MX [23] plus a scattered electron in the zero LL, labeled $X_{10}+e_{0}$. Moreover, there is a band above each free LL (labeled $2 e+h_{0}$ in Fig. 1 ) originating from the bound internal motion of two $2 \mathrm{D}$ electrons in $B$ (cf. [17]). Bound $X^{-}$states lie outside the continua. In the strictly-2D high- $B$ limit the only family of bound $X^{-}$states in zero LL's is the triplet $X_{t 00}^{-}$. There are no bound singlet $X_{s}^{-}$states [9,10] in contrast to the $B=0$ case. The obtained $X_{t 00}^{-}$binding energy $0.043 E_{0}$ is in agreement with [9.10]. In the next electron LL there are no bound singlet $X_{s}^{-}$states, and only one family of bound triplet states $X_{t 10}^{-}$. The $X_{t 10}^{-}$binding energy is $0.086 E_{0}$, twice that of the $X_{t 00}^{-}$. This is due to the fact that the two electrons in the triplet $X_{t 10}^{-}$state can occupy the single-particle states with zero $e^{-} h$ relative angular momenta $1 s$ (zero LL) and $2 s$ (first LL). This enhances the $e-h$ attraction relative to the ground $X_{t 00}^{-}$state in which electrons can occupy an antisymmetric combination of the $1 s$ and $2 p^{-}$single-particle states in zero LL.

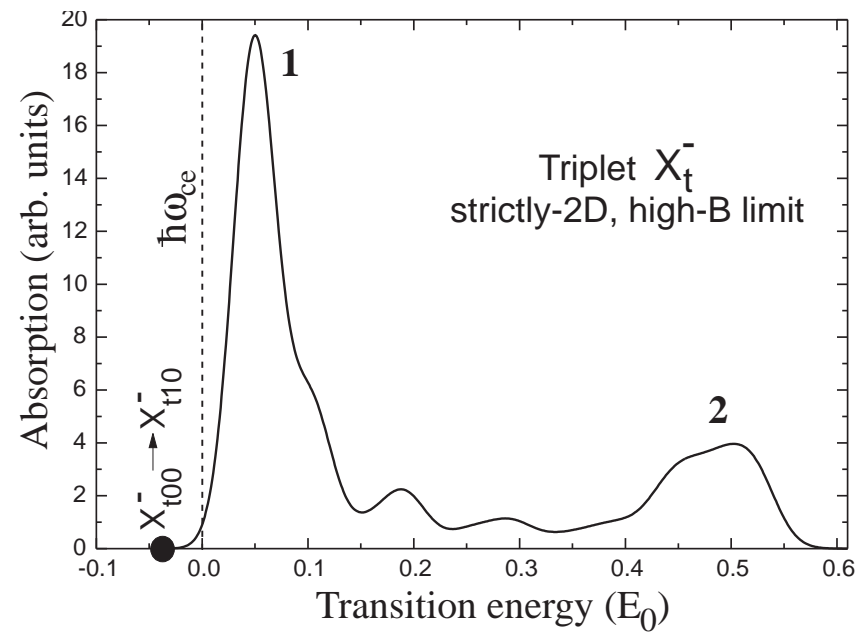

FIG. 2. Energies (in units of $E_{0}=\sqrt{\pi / 2} e^{2} / \epsilon l_{B}$, counted from $\left.\hbar \omega_{\text {ce }}\right)$ and dipole matrix elements of the internal transitions corresponding to Fig. 1. The filled dot shows the position of the forbidden $X_{t 00}^{-} \rightarrow X_{t 10}^{-}$transition. Spectra have been convoluted with the Gaussian of the $0.02 E_{0}$ width.

We first discuss internal $X^{-}$triplet transitions. In the $\sigma^{+}$polarization the inter-LL $\Delta N_{e}=1$ transitions are strong and gain strength with $B$ : Transitions with $\Delta N_{e} \neq 1$ are only due to LL mixing and weak as $\left[E_{0} / \hbar \omega_{\text {ce }(\mathrm{h})}\right]^{2} \sim B^{-1}$. Both bound-to-bound $X_{t 00}^{-} \rightarrow$ $X_{t 10}^{-}$and photoionizing bound-to-continuum transitions are possible. For the latter, due to the rich structure of the continuum, two exact selection rules (1i) are easily simultaneously satisfied. As a result, the photoionizing absorption spectra have intrinsic linewidth $\sim E_{0}$ with two prominent peaks above the $e-\mathrm{CR}$ (Fig.2). These peaks are associated with high densities of states at the edges of the two MX bands indicated in Fig. 1. Transitions to the $2 e+h_{0}$ band have extremely small oscillator strengths. Most of these qualitative features of photoionizing transitions are preserved at finite fields and confinement, where both the triplet and singlet bound $X^{-}$states exist. This has been shown by high-accuracy calculations for realistic GaAs/GaAlAs quantum wells at $B>8 \mathrm{~T}$, which are confirmed in recent experiments and will be reported elsewhere [24]. Here we are interested in the bound-to-bound $X_{t 00}^{-} \rightarrow X_{t 10}^{-}$transition. Note first that since the final state is more strongly bound, this transition has a specific spectral position - it lies below the $e-\mathrm{CR}$ energy $\hbar \omega_{\mathrm{ce}}$. However, in a translationally invariant system it is strictly prohibited. Indeed, the $X_{t 00}$ PS (with $k=0$ ) has $M_{z}=-1$, while the $X_{t 10}$ PS has $M_{z}^{\prime}=1$ (Fig. 1). It follows then that the selection rules (位) $\Delta k=0$ and $\Delta M_{z}=1$ cannot be simultaneously satisfied. This also holds at finite $B>8 \mathrm{~T}$ and in quasi-2D quantum wells [24]. Localization of charged excitons breaks translational invariance and relaxes selection rules. As a result, the bound-to-bound $X_{t 00}^{-} \rightarrow X_{t 10}^{-}$ transition develops below the $e^{-\mathrm{CR}}$. Such a peak is a telltale mark of localization of charged triplet excitons. The 
strong triplet $T^{-}$transition of the $D^{-}$center (two electrons bound by a donor ion), which was predicted theoretically 21] and observed experimentally [25], can be thought of as one of the possible limiting cases, namely, when the hole is completely localized.

Consider now photoluminescence (PL) from the triplet ground state $X_{t 00}^{-} \rightarrow$ photon $+e_{n}^{-}$with the electron in the $n$-th LL in the final state; $n=1,2, \ldots$ correspond to shake-up processes in the PL [3] 5 . The PL selection rules are $\Delta k=0$ and $\Delta M_{z}=0$. The triplet $X_{t 00}^{-}$ground PS with $k=0$ has $M_{z}=-1$ (also at finite $B>8 \mathrm{~T}$ and in quasi-2D quantum wells [10,24), while the electron in the $n$-th LL, with the factored wave function $\phi_{n m}^{(e)}$, has $m_{z}=n-m$. The corresponding optical matrix element for transition to any LL $n \geq 0$ is zero: $\left\langle\phi_{n m}^{(e)}\left|\hat{\mathcal{L}}_{\mathrm{PL}}\right| X_{t 00\left(M_{z}=-1, k=0\right)}^{-}\right\rangle \sim \delta_{m, k=0} \delta_{n-m,-1}$. This means that the ground triplet state of an isolated $X_{t}^{-}$is optically inactive - dark in PL. In the strictly2D high- $B$ limit this also follows [9] from the "hidden symmetry" in $e-h$ systems 26]. Our result is much more general. Indeed, as discussed above, quasi-2D effects, $e-h$ asymmetry, admixture of higher LL's, and the complex character of the valence band break neither axial nor translational symmetry. Therefore, even in the presence of these effects, the triplet stays dark - as long as the ground $X_{t}^{-}$PS has $M_{z}<0$. This exact result was overlooked in [9,10]; very small but finite $X_{t}^{-}$oscillator strengths obtained in [9,10] are in fact artifacts coming from finite-size calculations. Note that the quasi-2D $X_{s}^{-}$ singlet ground PS has $M_{z}=0$ [10,11,24 and is optically active in PL: $\left\langle\phi_{n m}^{(e)}\left|\hat{\mathcal{L}}_{\mathrm{PL}}\right| X_{s 00\left(M_{z}=0, k=0\right)}^{-}\right\rangle \sim \delta_{m, 0} \delta_{n, 0}$. We see, however, that the shake-up processes are prohibited in PL from the isolated singlet ground state $X_{s}^{-}$. The question now remains why in fact the $X_{t}^{-}$ground state is visible in experiment in $B$ [1] and the singlet $X_{s}^{-}$ shake-up processes are commonly observed in PL - even at very low densities of excess free carriers [3,5]? Our results show that there should be mechanisms breaking the underlying exact translational and rotational symmetries. We interpret this as an indication toward localization of charged excitons in $B$. More theoretical and experimental work is needed here to establish, in particular, the regime of localization of charged excitons.

In conclusion, we have shown that due to magnetic translations, dipole-allowed transitions of charged mobile semiconductor complexes are governed in magnetic fields $B$ by a novel exact selection rule. Some experimentally observed features in interband photoluminescence of quasi-2D charged excitons $X^{-}$in $B$ cannot be explained without accounting for symmetry-breaking effects, an indication toward localization. The appearance of the peak below the electron cyclotron resonance, corresponding to the internal bound-to-bound $X^{-}$triplet transition, is a characteristic mark associated with breaking of translational invariance. We propose using this as a tool for studying the extent of $X^{-}$localization.

We thank I. Bar-Joseph, B.D. McCombe, D.M. Whittaker, and D.R. Yakovlev for useful discussions. ABD is grateful to the Humboldt Foundation for research support.

* on leave from General Physics Institute, RAS, Moscow 117942, Russia.

[1] G. Finkelstein, H. Shtrikman, and I. Bar-Joseph, Phys. Rev. Lett. 74, 976 (1995); S. Glasberg et al., Phys. Rev. B 59, R10 425 (1999).

[2] A. J. Shields et al., Phys. Rev. B 52, 7841 (1995).

[3] G. Finkelstein, H. Shtrikman, and I. Bar-Joseph, Phys. Rev. B 53, 12593 (1996); 56, 10326 (1997).

[4] D. R. Yakovlev et al., Phys. Rev. Lett. 79, 3974 (1997).

[5] O. V. Volkov et al., JETP Lett. 67, 744 (1998).

[6] G. Eytan et al., Phys. Rev. Lett. 81, 1666 (1998); G. Finkelstein et al., Phys. Rev. B 58, 12637 (1998); H. Okamura et al., Phys. Rev. B 58, R15 985 (1998).

[7] M. Hayne et al., Phys. Rev. B 59, 2927 (1999).

[8] A. Wójs and P. Hawrylak, Phys. Rev. B 51, 10880 (1995).

[9] J. J. Palacios, D. Yoshioka, and A. H. MacDonald, Phys. Rev. B 54, R2296 (1996).

[10] D. M. Whittaker and A. J. Shields, Phys. Rev. B 56, 15185 (1997).

[11] B. Stebe et al., Phys. Rev. B 58, 9926 (1998).

[12] J. J. Quinn et al., cond-mat/9905323.

[13] W. Kohn, Phys. Rev. 123, 1242 (1961).

[14] L. P. Gor'kov and I. E. Dzyaloshinskii, Sov. Phys. JETP 26, 449 (1968).

[15] J. E. Avron, I. W. Herbst, and B. Simon, Annals of Phys. 114, 431 (1978).

[16] B. R. Johnson, J. O. Hirschfelder, and Kuo-Ho Yang, Rev. Mod. Phys. 55, 109 (1983).

[17] S. A. Trugman and S. Kivelson, Phys. Rev. B 31, 5280 (1985).

[18] For an electron system in $B$ (with $e_{i} / m_{i}=$ const), the Kohn theorem $\left[H, \hat{V}^{ \pm}\right]= \pm \hbar \omega_{\mathrm{ce}} \hat{V}^{ \pm}$holds 13]. Due to the CM separation, both conservation of $k$ and the Kohn theorem - though based on different operator algebras - give in this case equivalent results.

[19] H. Haug and S. W. Koch, Quantum theory of the optical and electronic properties of semiconductors (World Scientific, Singapore, 1993).

[20] In the high- $B$ limit, the $X^{+}$eigenspectra are obtained from those of $X^{-}$with the substitution $M_{z} \rightarrow-M_{z}$.

[21] A. B. Dzyubenko, Phys. Lett. A 165, 357 (1992); A. B. Dzyubenko and A. Yu. Sivachenko, Phys. Rev. B 48, 14690 (1993).

[22] A. B. Dzyubenko, Phys. Lett. A 173, 311 (1993).

[23] I. V. Lerner and Yu. E. Lozovik, Sov. Phys. JETP 51, 588 (1980).

[24] H. A. Nickel et al., to be published.

[25] R. S. Ryu et al., Phys. Rev. B 54, R11 086 (1996).

[26] I. V. Lerner and Yu. E. Lozovik, Sov. Phys. JETP 53, 763 (1981); A. B. Dzyubenko and Yu. E. Lozovik, Sov. Phys. Solid State 25, 874 (1983); 26, 938 (1984); J. Phys. A 24, 415 (1991); A. H. MacDonald and E. H. Rezayi, Phys. Rev. B 42, 3224 (1990). 